Pacific Journal of Mathematic 


\section{UNIFORMIZABLE SPACES WITH}

A UNIQUE STRUCTURE

\section{S. GÁL}

Here we shall study only uniformizable Hausdorff spaces. In general if a topological space $X$ is uniformizable then there are many uniform structures 2 compatible with the topology of $X$. If $X$ is compact then there is only one uniform structure for $X$ and there are also non-compact spaces whose structures are uniquely determined by their topology. (See [1] and [2].) The purpose of this note is to give a necessary and sufficient condition that $\mathscr{Z}$ be uniquely determined by $X$. Let $C(X)$ be the algebra of bounded real valued continuous functions on $X$ and let $C(X)$ be topologized by the topology of uniform convergence on the whole space $X$. By $A(X)$ we denote the subalgebra of those real valued continuous functions which are constant on the complement of some compact set in $X$. We shall prove the following

THEOREM. The uniformizable Hausdorff space $X$ admits only one uniform structure if and only if $A(X)$ is dense in $C(X)$.

Another necessary and sufficient condition for uniqueness was found earlier by $R$. Doss [3]: The closed sets $C_{1}$ and $C_{2}$ are called normally separable if there exists a continuous real valued function $f$ on $X$ which takes the value 1 on $C_{1}$ and the value 2 on $C_{2}$. Doss proved the following:

Uniqueness takes place if and only if of any two normally separable sets at least one is compact.

The following proof of the Theorem makes no use of this criterion given by Doss. However at the end it will be proved that the criterion stated in the Theorem and the criterion due to Doss are equivalent. This gives a new, simpler proof of Doss's theorem. Approximately at the same time when [3] was published P. Samuel in [5] and T. Shirota in [6] proved that

Among the uniform structures compatible with the topology of $X$ there is a weakest if and only if $X$ is locally compact.

The two halfs of this theorem are stated as of Lemma 3 and Lemma 6 below. Their proofs are independent of the rest of the paper and so they furnish a simple proof for the Samuel-Shirota theorem.

A space $X$ is said to be normally imbedded in the space $Y$ if every real valued continuous function on $X$ admits a continuous extension to $Y$. If this property is supposed to hold only for bounded functions one speaks about a bounded normal imbedding. E. Hewitt in [4] proved that

Received December 24, 1958. 
The Hausdorff space $X$ is normally imbedded in every uniformizable space containing $X$ as a dense subspace if and only if of any two disjoint sets at least one is compact.

Among all uniform structures compatible with the topology of a uniformizable space $X$ there is a strongest called the Weil structure or the universal structure of $X$. Its existence follows from the fact that the union of all uniform structures compatible with $X$ is a subbase for a uniform structure which is compatible with $X$. The Weil structure $\mathscr{Q}_{W}$ is uniquely determined by the following property: If $\mathscr{V}$ is a uniform structure for $Y$ and $f: X \rightarrow Y$ is continuous with respect to the topology of $X$ and the uniform topology associated with $\mathscr{Y}$ then $f$ is uniformly continuous with respect to $\mathscr{U}_{W}$ and $\mathscr{V}^{.}$. In general $\mathscr{U}_{W}$ is not a precompact structure.

Let $X$ satisfy the criterion given by Doss and let $\mathscr{U}$ be the unique structure compatible with its topology. The uniqueness implies that $\mathscr{U}$ is identical with the Weil structure of $X$. Let $X$ be a dense subspace of the uniformizable space $Y$ and let $\mathscr{V}$ be a uniform structure for $Y$. The restriction of $\mathscr{Y}$ to $X$ is the Weil structure of $X$ and so every real valued continuous function $f$ on $X$ is uniformly continuous with respect to $\mathscr{V}^{\wedge}$. Consequently $f$ can be extended to a uniformly continuous function on $Y$ and so $X$ is normally imbedded in $Y$. Thus by Hewitt's theorem one of any two disjoint closed sets of $X$ must be compact. Combining the present Theorem with the theorms of Doss and Hewitt we obtain:

Any two of the following statements are equivalent:

(i) $X$ has a unique uniform structure.

(ii) If $C_{1}$ and $C_{2}$ are normally separable closed sets in $X$ then at least one of them is compact.

(iii) If $C_{1}$ and $C_{2}$ are disjoint closed sets in $X$ then at least one of them is compact.

(iv) $A(X)$ is dense in $C(X)$.

(v) $X$ is normally imbedded in every uniformizable space containing $X$ as a dense subspace.

We omitted the analogue of (v) concerning bounded normal imbeddings. For we have:

If $X$ has a unique uniform structure then every real valued continuous function is bounded on $X$.

This follows from Lemma 1 below. Using (iii) one can also prove that any two disjoint closed sets are normally separable.

The following notations will be used: Open sets will be denoted by $O$, closed sets by $C$, neighborhoods by $N_{x}$ and open neighborhoods by $O_{x}$. For the closure of a set $A$ we write $\bar{A}$ and $c A$ stands for the complement of $A$ with respect to a given set containing $A$. Uniform structures will be denoted by $\mathscr{U}, \mathscr{V}, \ldots$; the completion of a uniform space 
$X$ with respect to a structure $\mathscr{U}$ will be denoted by $\bar{X}$ and the complete structure will be denoted by $\overline{\mathscr{U}}$. As usual $U \circ V$ is the composition of the vicinities $U, V \in \mathscr{U}$ and $U[x]=[y:(x, y) \in U]$. If $\mathscr{U}_{i}(i \in I)$ are uniform structures for $X$ then lub $\mathscr{Q}_{i}$ denotes the uniform structure generated by the subbase $\cup \mathscr{U}_{i}$. It is the weakest structure which is stronger than any $\mathscr{U}_{i}(i \in I)$. If $f$ is uniformly continuous on $X$ then $\bar{f}$ denotes its extension to $\bar{X}$. The structure $\mathscr{Z}_{0}$ used in the proof of Lemma 5 is the so-called Cech structure which was introduced by Samuel in [5]. The fact that the definition given in [5] is equivalent to the present simpler definition follows from Lemma 4 . $\mathscr{U}_{0}$ is the strongest precompact structure compatible with the topology of $X$ and its completion is the Stone-Čech compactification $\beta X$.

Lemma 1. If $A(X)$ is dense in $C(X)$ then every uniform structure $\mathscr{U}$ compatible with the topology of $X$ is precompact.

Proof. This follows by a simple argument which is used also in [3]: Suppose that $X$ is a topological space and $\mathscr{Q}$ is a non-precompact structure compatible with the topology of $X$. Then there is a symmetric vicinity $U \in \mathscr{U}$ and a sequence of points $x_{1}, x_{2}, \ldots$ in $X$ such that $\left(x_{m}, x_{n}\right) \in U$ only if $m=n$. We choose a symmetric $V \in \mathscr{V}$ satisfying $V \circ V \subseteq U$ and a symmetric $W \in \mathscr{U}$ satisfying $W \circ W \subseteq V$. Since $X$ is completely regular there is a real valued continuous function $f_{n}$ on $X$ with the property that $\left|f_{n}(x)\right| \leq 1$ for every $x \in X$, the closure of $W\left[x_{n}\right]$ is a support of $f_{n}$ and $f_{n}(x)= \pm 1$ according as $n$ is even or odd. By $W \circ W \subseteq V$ the closure of $W\left[x_{n}\right]$ is contained in $V\left[x_{n}\right]$ and by $V \circ V \subseteq U$ the sets $V\left[x_{m}\right]$ and $V\left[x_{n}\right]$ intersect only if $m=n$. Therefore the series $\Sigma f_{n}(x)$ contains for each $x \in X$ at most one nonvanishing term and it defines a bounded continuous function $f$ on $X$. Neither $\left\{x_{1}, x_{3}, \cdots\right\}$ nor $\left\{x_{2}, x_{4}, \cdots\right\}$ is compact and so $f$ can not be approximated uniformly on $X$ by elements of $A(X)$. Hence the existence of non-precompact structure implies that $A(X)$ is not dense in $C(X)$.

\section{Lemma 2. If $A(X)$ is dense in $C(X)$ then $X$ is locally compact.}

Proof. Let $O_{x}$ be an open neighborhood of the point $x \in X$ and let $f$ be a real valued continuous function on $X$ such that $0 \leq f(\xi) \leq 1$ for every $\xi \in X, f(x)=1$ and $f(\xi)=0$ if $\xi \notin O_{x}$. Since $A(X)$ in dense in $C(X)$ there is a continuous function $g$ which is constant on the complement $O$ of a compact set $C$ and is such that $|f(\xi)-g(\xi)| \leq \varepsilon$ for every $\xi \in X$. If $N_{x}=[\xi: f(\xi) \geq 1-\varepsilon]$ is a subset of $C$ then $N_{x}$ is a compact neighborhood of $x$. If this is not the case then $O$ and $N_{x}$ have a common 
point $\xi$. Then for every $\eta \in O$ we have

$$
f(\eta) \geq g(\eta)-\varepsilon=g(\xi)-\varepsilon \geq f(\xi)-2 \varepsilon \geq 1-3 \varepsilon>0
$$

and so $\eta \in O_{x}$. Since $O \subseteq O_{x}$ where $C=c O$ is compact we see that the complement of $O_{x}$ is compact. If this is the situation for every open neighborhood $O_{x}$ of $x$ then $X$ is compact. Hence either $N_{x}$ is a compact neighborhood of $x$ for every $x \in X$ or $X$ is a compact space.

Let $f$ map $X$ into $Y$ and let $\mathscr{Y}$ be a uniform structure for $Y$. The sets $f^{-1}(V)=\left[\left(x_{1}, x_{2}\right):\left(f\left(x_{1}\right), f\left(x_{2}\right)\right) \in V\right](V \in \mathscr{V})$ form a base for a uniform structure $\mathscr{U}$ for $X$, called the inverse image of $\mathscr{V}$ under $f$. If $f$ is a real valued function on $X$ and $\mathscr{V}$ is the usual structure of the reals the inverse structure will be denoted by $\mathscr{U}_{f}$. It is a pseudo-metric structure which is generated by the pseudo-metric $d_{f}\left(x_{1}, x_{2}\right)=\left|f\left(x_{1}\right)-f\left(x_{2}\right)\right|$. If $f$ is bounded then $\mathscr{Q}_{f}$ is precompact. If $\{f\}$ is a family of real valued functions on $X$ we call lub $\mathscr{U}_{f}$ the uniform structure generated by the family $\{f\}$. Every $f$ in $\{f\}$ is uniformly continuous with respect to lub $\mathscr{U}_{f}$. Moreover if $\mathscr{U}$ is a uniform structure for $X$ and if every $f$ in $\{f\}$ is uniformly continuous with respect to $\mathscr{U}$ then lub $\mathscr{U}_{s} \leq \mathscr{U}$. If every $f \in\{f\}$ is bounded then lub $\mathscr{U}_{f}$ is a precompact structure for $X$. These simple consequences are presented in greater detail in Chapter IX of [1].

Some interesting uniform structures are structures generated by families of real valued functions $\{f\}$. For example let $X$ be locally compact and let $\{f\}$ be the family $A(X)$. Given $x \in X$ and a compact neighborhood $C_{x}$ of $x$ there is a real valued continuous function $f$ on $X$ such that $f(x)=1$ and $C_{x}$ is a support of $f$. Hence $C_{x}$ is a neighborhood of $x$ in the uniform topology associated with $\mathscr{U}_{f}$. It follows that $\mathscr{U}=$ lub $\mathscr{U}_{\rho}$ is compatible with the topology of $X$. Every $f \in A(X)$ is constant on the complement of a compact set and so it is uniformly continuous with respect to any uniform structure $\mathscr{V}$ which is compatible with $X$. Therefore $\mathscr{U} \leq \mathscr{V}$ and so $\mathscr{U}$ is the weakest structure compatible with the topology given on $X$. Hence we proved the following lemma, which incidentally is an exercise in [1]. (See Chap. IX. p. 16 Exercise 11.)

Lemma 3. If $X$ is a locally compact Hausdorff space then there is a weakest uniform structure which is compatible with the topology of $X$. It is the uniform structure generated by the family $A(X)$.

The weakest structure if it exists is necessarily precompact. Now we show that every precompact separated structure can be generated by families of real valued functions. For let $\mathscr{U}$ be a precompact separated structure for $X$ and let $\bar{X}$ be the completion of $X$ with respect to $\mathscr{U}$. The completed structure will be denoted by $\overline{\mathscr{U}}$. Let $\mathscr{U}_{\bar{s}}$ denote the 
uniform structure generated on $\bar{X}$ by the real valued function $\bar{f}$ given on $\bar{X}$. It is clear that the restriction of $\mathscr{U}_{\bar{f}}$ to $X$ is the same as the structure $\mathscr{U}_{f}$ generated on $X$ by the restriction $f$ of $\bar{f}$ to $X$. More generally if $\{\bar{f}\}$ is a family of real valued functions on $\bar{X}$ then the restriction of lub $\mathscr{U}_{\bar{f}}$ to $X$ is the structure lub $\mathscr{Q}_{f}$. If $\{\bar{f}\}$ is the family of all real valued continuous functions on $\bar{X}$ then lub $\mathscr{C}_{\bar{f}}$ is compatible with the topology of $\bar{X}$ and so by the compactness of $\bar{X}$ we have $\overline{\mathscr{U}}_{\mathscr{C}}=$ lub $\mathscr{U}_{\bar{f}}$. Therefore $\mathscr{U}=$ lub $\mathscr{U}_{f}$ where $\{f\}$ is the family of the restrictions of continuous functions $\bar{f}$ to $X$. Since $f$ is the restriction of some $\bar{f}$ if and only if $f$ is uniformly continuous with respect to $\mathscr{U}$ we have

LEMMA 4. Every precompact separated structure $\mathscr{U}$ is generated by the family of those real valued functions which are uniformly continuous with respect to $\mathscr{U}$.

The topology of uniform convergence on $X$ is meaningful on the linear space $L$ of all real valued functions on the set $X$ : The $\varepsilon$-neighborhood of 0 consists of those functions $f$ on $X$ for which supr $|f(x)|<\varepsilon$. Let $A, C \subseteq L$ and let $A$ be dense relative to $C$. By $\mathscr{U}_{A}$ and $\mathscr{U}_{C}$ we denote the uniform strutures generated by the families $A$ and $C$, respectively. Then for every $c \in C$ and $\varepsilon>0$ there is an $a \in A$ such that $|a(x)-c(x)|<\varepsilon / 4$ for every $x \in X$ and so

$$
[(x, y):|c(x)-c(y)|<\varepsilon] \supseteq\left[(x, y):|a(x)-a(y)|<\frac{\varepsilon}{2}\right]
$$

This implies that every vicinity of $\mathscr{U}_{0}$ contains a vicinity of $\mathscr{U}_{0}$ so that $\mathscr{U}_{C} \leq \mathscr{\ell}_{A}$. If in addition $A \subseteq C$ then $\mathscr{U}_{A} \leq \mathscr{U}_{O}$ and so we have

Lemma 5. If $A$ is dense in $C$ then they generate the same uniform structure.

Now it is easy to show that if $A(X)$ is dense in $C(X)$ then there is only one uniform structure which is compatible with the topology of $X$ : By Lemma 2 the space $X$ is locally compact and so by Lemma 3 it has a weakest uniform structure $\mathscr{C}_{w}$ which is compatible with its topology. By the same lemma $\mathscr{U}_{w}$ is generated by $A(X)$. It will be sufficient to show that $\mathscr{U}_{w}$ is identical with the Weil structure $\mathscr{U}_{W}$ of $X$. By Lemma $1 \mathscr{U}_{W}$ is precompact and so by Lemma 4 it is generated by the family of those real valued functions on $X$ which are uniformly continuous with respect to $\mathscr{Z}_{W}$. By the precompactness and by the definition of $\mathscr{C}_{W}$ this family is $C(X)$. Since $A(X)$ is dense in $C(X)$ by Lemma 5 they generate the same structure, that is $\mathscr{U}_{w}=\mathscr{U}_{w}$. This 
proves the sufficiency of the condition given in the Theorem.

Now we shall prove that the condition stated in the Theorem is also necessary. First we suppose that $X$ is a locally compact Hausdorff space. Let $\bar{X}=X \cup\{\infty\}$ be the Alexandroff compactification of $X$ and let $\mathscr{U}_{A}$ be the uniform structure obtained for $X$ by restricting the unique structure of $\bar{X}$ to $X$. We prove that a real valued function $f$ is uniformly continuous with respect to $\mathscr{C}_{A}$ if and only if $f$ belongs to the uniform closure of $A(X)$. For compact $X$ this is obvious so we may assume that $X$ is not a compact space. Since the elements of $A(X)$ are uniformly continuous with respect to any structure compatible with the topology of $X$ the same holds for the elements of its closure $\overline{A(X)}$ and so it will be sufficient to show that if $f$ is uniformly continuous with respect to $\mathscr{U}_{A}$ then $f \in \overline{A(X)}$. However if $f$ is uniformly continuous with respect to $\mathscr{U}_{A}$ then it has a continuous extension $\bar{f}$ to $\bar{X}$. By the continuity of $\bar{f}$ at $\infty$ for every $\varepsilon>0$ there is a compact set $C \subset X$ such that $|\bar{f}(x)-\bar{f}(\infty)|<\varepsilon$ for every $x \notin C$. Let $O$ be an open neighborhood of $C$ which does not contain $\infty$. Since $\bar{X}$ is normal there is a real valued continuous function $\bar{g}$ on $\bar{X}$ which takes the value 1 on $C$, vanishes outside of $O$ and satisfies $0 \leq g(x) \leq 1$ on $\bar{X}$. Then $h=(f-\bar{f}(\infty)) g+$ $\bar{f}(\infty)$ belongs to $A(X)$ and is such that $|h(x)-f(x)|<\varepsilon$ for every $x \in X$.

Let us now suppose that $A(X)$ is not dense in $C(X)$. Then there is an $f \in C(X)$ which is not in $\overline{A(X)}$ and so it is not uniformly continuous with respect to $\mathscr{U}_{A}$. Since every element of $C(X)$ is uniformly continuous with respect to the uniform structure $\mathscr{U}_{\sigma}$ generated by $C(X)$ we see that $\mathscr{U}_{A}$ and $\mathscr{U}_{O}$ are distinct structures compatible with the topology of $X$. This proves the necessity of the condition in the case of locally compact spaces.

The proof of the Theorem will be completed by showing

Lemma 6. If the uniformizable Hausdorff space $X$ is not locally compact then there is no weakest among the uniform structures which are compatible with the topology of $X$.

Proof. First we notice that if $X$ is a Hausdorff space and if the Hausdorff space $\bar{X}$ is a compactification of $X$ which contains only finitely many more elements than $X$ then $X$ is locally compact. Now let $\mathscr{U}$ be a uniform structure which is compatible with the topology of the uniformizable Hausdorff space $X$. We assume that $X$ is not locally compact. Let $B$ denote the family of those bounded real valued functions on $X$ which are uniformly continuous with respect to $\mathscr{U}$ and let $\mathscr{V}$ be the uniform structure generated on $X$ by $B$. Then $\mathscr{V}$ is precompact and is not stronger than $\mathscr{U}$. Let $\bar{X}$ be the compact comple- 
tion of $X$ with respect to $\mathscr{Y}$. By the foregoing remark $\bar{X}-X$ is an infinite set. We consider the space $\bar{Y}$ obtained from $\bar{X}$ by identifying a finite number of distinct points $x_{1}, \cdots, x_{n}(n>1)$ of $\bar{X}-X$. The identification space $\bar{Y}$ will be compact and separated, so it has a unique uniform structure whose restriction to $X$ will be denoted by $\mathscr{\mathscr { V }}$. Then $\bar{Y}$ is the completion of $X$ with respect to $\mathscr{W}$ and $\bar{X}$ is the completion of $X$ with respect to $\mathscr{V}$. By Lemma 4 both $\mathscr{Y}$ and $\mathscr{W}$ are generated by their families of real valued uniformly continuous functions. A real valued function is uniformly continuous with respect to $\mathscr{W}$ if and only if it is uniformly continuous with respect to $\mathscr{Y}$ and its extension to $\bar{X}$ assumes the same value at $x_{1}, \cdots, x_{n}$. Hence $\bar{X}$ being separated there are real valued functions on $X$ which are uniformly continuous with respect to $\mathscr{V}^{\prime}$ but not with respect to $\mathscr{W}$. Therefore $\mathscr{W}<\mathscr{Y} \leq \mathscr{U}$ and so $X$ has no weakest structure compatible with its topology. Lemma 6 and the Theorem are proved.

We finish by proving that the condition given in the Theorem is equivalent to the condition of Doss. First suppose that $A(X)$ is dense in $C(X)$. Let $C_{0}$ and $C_{1}$ be normally separated by $f$. We may assume that $0 \leq f(x) \leq 1$ for every $x \in X, f$ is 0 on $C_{0}$ and 1 on $C_{1}$. We choose a $g \in A(X)$ satisfying $|f(x)-g(x)|<\varepsilon<\frac{1}{2}$ everywhere on $X$. Let $g$ be constant on the complement of the compact set $C$. If this constant value is neither 0 nor 1 then both $C_{0}$ and $C_{1}$ are compact. Otherwise we may restrict ourselves to the case when $C$ is a compact support of $g$. If $x \notin C$ then $g(x)=0$ so $f(x)<\varepsilon$ and $x \in C_{0 \text { ₹ }}=[x: f(x) \leq \varepsilon]$. Therefore $c C \subseteq C_{0 \Xi} \subseteq c C_{1 \varepsilon}=[x: f(x)<1-\varepsilon]$. This shows that $C_{1}=[x: f(x)=1] \subseteq C$ and so $C_{1}$ is compact.

Next we suppose that $X$ satisfies Doss's condition. Let $f \in C(X)$ and $\varepsilon>0$ be given. We consider the closed sets $C_{k}=\left[x:|f(x)-k \varepsilon| \leq \frac{1}{2}\right]$ where $k=0, \pm 1, \pm 2, \cdots$. Their union is $X$. Any two of the sets $C_{2 k}(k=0, \pm 1, \pm 2, \cdots)$ are normally separable so at most one of them is not compact. Similarly at most one of the sets $C_{2 k+1}(k=0, \pm 1, \pm 2, \cdots)$ can be non-compact. Moreover if $C_{2 k}$ and $C_{2 l+1}$ are not compact they must have common points and so

$$
C_{2 k} \cup C_{2 l+1}=\left[x: \varepsilon\left(m-\frac{1}{2}\right) \leq f(x) \leq \varepsilon\left(m+\frac{3}{2}\right)\right]
$$

for some $m$. We define

$$
g(x)= \begin{cases}f(x) & \text { if } x \in C_{k} \text { and } k<m \\ \varepsilon\left(m-\frac{1}{2}\right) & \text { if } x \in C_{2 k} \cup C_{2 l+1} \\ f(x)-\varepsilon & \text { if } x \in C_{k} \text { and } k>m+1 .\end{cases}
$$

Then $|f(x)-g(x)| \leq \varepsilon$ for every $x \in X$ and $g \in A(X)$ because $f$ being 
bounded there are only finitely many non-void sets among the sets $C_{k}$. If only $C_{2 k}$ or only $C_{2 l+1}$ is non-compact the construction is similar.

\section{REFERENCES}

1. N. Bourbaki, Topologie générale, Chap. I, II et IX; 2nd edition Hermann and Cie, Editeurs Paris (1951) and (1958).

2. J. Dieudonné, Sur les espaces uniformes complets, Ann. Ec. Normal Sup. 3e série 56 (1939), 277-291.

3. R. Doss, On uniform spaces with a unique structure, Amer. J. Math. 71 (1949), 19-23.

4. E. Hewitt, A note on extensions of continuous real functions, Assais Acad. Brasil Ci. 21 (1949), 175-179.

5. P. Samuel, Ultrafilters and compactification of uniform spaces, Trans. Amer. Math. Soc. 64 (1948), 100-132.

6. T. Shirota, On systems of structures of a completely regular space, Osaka Math. Journal 2 (1950), 131-143.

YALE UNIVERSITY AND CORNELL UNIVERSITY 


\section{PACIFIC JOURNAL OF MATHEMATICS}

EDITORS

David Gilbarg

Stanford University

Stanford, California

R. A. Beaumont

University of Washington

Seattle 5 , Washington
A. L. Whiteman

University of Southern California

Los Angeles 7, California

L. J. PAIGE

University of California

Los Angeles 24, California

\section{ASSOCIATE EDITORS}

\author{
E. F. BECKENBACH \\ C. E. BURGESS \\ E. HEWITT \\ A. HORN
}

\author{
V. GANAPATHY IYER \\ R. D. JAMES \\ M. S. KNEBELMAN \\ L. NACHBIN
}
I. NIVEN
E. G. STRAUS
T. G. OSTROM
G. SZEKERES
H. L. ROYDEN
F. WOLF
M. M. SCHIFFER
K. YOSIDA

\section{SUPPORTING INSTITUTIONS}

\author{
UNIVERSITY OF BRITISH COLUMBIA \\ CALIFORNIA INSTITUTE OF TECHNOLOGY \\ UNIVERSITY OF CALIFORNIA \\ MONTANA STATE UNIVERSITY \\ UNIVERSITY OF NEVADA \\ OREGON STATE COLLEGE \\ UNIVERSITY OF OREGON \\ OSAKA UNIVERSITY \\ UNIVERSITY OF SOUTHERN CALIFORNIA
}

\author{
STANFORD UNIVERSITY \\ UNIVERSITY OF TOKYO \\ UNIVERSITY OF UTAH \\ WASHINGTON STATE COLLEGE \\ UNIVERSITY OF WASHINGTON \\ $* \quad * \quad *$ \\ AMERICAN MATHEMATICAL SOCIETY \\ CALIFORNIA RESEARCH CORPORATION \\ HUGHES AIRCRAFT COMPANY \\ SPACE TECHNOLOGY LABORATORIES
}

Mathematical papers intended for publication in the Pacific Journal of Mathematics should be typewritten (double spaced), and the author should keep a complete copy. Manuscripts may be sent to any one of the four editors. All other communications to the editors should be addressed to the managing editor, L. J. Paige at the University of California, Los Angeles 24, California.

50 reprints per author of each article are furnished free of charge; additional copies may be obtained at cost in multiples of 50 .

The Pacific Journal of Mathematics is published quarterly, in March, June, September, and December. The price per volume (4 numbers) is $\$ 12.00$; single issues, $\$ 3.50$. Back numbers are available. Special price to individual faculty members of supporting institutions and to individual members of the American Mathematical Society: $\$ 4.00$ per volume; single issues, $\$ 1.25$.

Subscriptions, orders for back numbers, and changes of address should be sent to Pacific Journal of Mathematics, 2120 Oxford Street, Berkeley 4, California.

Printed at Kokusai Bunken Insatsusha (International Academic Printing Co., Ltd.), No. 6, 2-chome, Fujimi-cho, Chiyoda-ku, Tokyo, Japan.

PUBLISHED BY PACIFIC JOURNAL OF MATHEMATICS, A NON-PROFIT CORPORATION

The Supporting Institutions listed above contribute to the cost of publication of this Journal, but they are not owners or publishers and have no responsibility for its content or policies. 


\section{Pacific Journal of Mathematics}

\section{Vol. 9, No. 4 \\ August, 1959}

Frank Herbert Brownell, III, A note on Kato's uniqueness criterion for

Schrödinger operator self-adjoint extensions ............... 953

Edmond Darrell Cashwell and C. J. Everett, The ring of number-theoretic

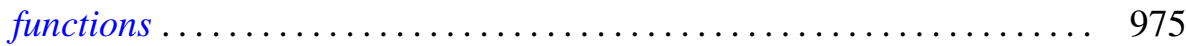

Heinz Otto Cordes, On continuation of boundary values for partial

differential operators ............................. 987

Philip C. Curtis, Jr., n-parameter families and best approximation . . . . . . 1013

Uri Fixman, Problems in spectral operators . . . . . . . . . . . . . . . 1029

I. S. Gál, Uniformizable spaces with a unique structure .............. 1053

John Mitchell Gary, Higher dimensional cyclic elements ............ 1061

Richard P. Gosselin, On Diophantine approximation and trigonometric

polynomials ..................................... 1071

Gilbert Helmberg, Generating sets of elements in compact groups ........ 1083

Daniel R. Hughes and John Griggs Thompson, The H-problem and the

structure of $H$-groups .................................. 1097

James Patrick Jans, Projective injective modules ................. 1103

Samuel Karlin and James L. McGregor, Coincidence properties of birth and

death processes ..................................... 1109

Samuel Karlin and James L. McGregor, Coincidence probabilities ........ 1141

J. L. Kelley, Measures on Boolean algebras ................... 1165

John G. Kemeny, Generalized random variables ................... 1179

Donald G. Malm, Concerning the cohomology ring of a sphere bundle ... . . 1191

Marvin David Marcus and Benjamin Nelson Moyls, Transformations on

tensor product spaces .................................. 1215

Charles Alan McCarthy, The nilpotent part of a spectral operator ........ 1223

Kotaro Oikawa, On a criterion for the weakness of an ideal boundary

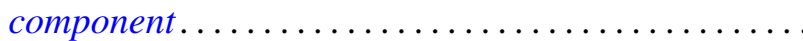

Barrett O'Neill, An algebraic criterion for immersion . . ............... 1239

Murray Harold Protter, Vibration of a nonhomogeneous membrane ... . . . . 1249

Victor Lenard Shapiro, Intrinsic operators in three-space . . . . . . . . . . . 1257

Morgan Ward, Tests for primality based on Sylvester's cyclotomic

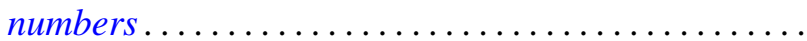

L. E. Ward, A fixed point theorem for chained spaces ....

Alfred B. Willcox, Šilov type $C$ algebras over a connected locally compact

abelian group..................................... 1279

Jacob Feldman, Correction to "Equivalence and perpendicularity of

Gaussian processes" ........................ 